\title{
CFHT/SITELLE Observations of the Ejected WR Nebula M1-67
}

\author{
N. St-Louis ${ }^{1}$, M. Sévigny ${ }^{2}$, L. Drissen ${ }^{2}$ and T. Martin ${ }^{2}$ \\ ${ }^{1}$ Département de physique, Université de Montréal \\ C.P. 6128, Succ. Centre Ville, Montréal (Qc), H3C 3J7, Canada \\ ${ }^{2}$ Département de physique, de génie physique et d'optique, \\ Université Laval, Québec (QC) G1V 0A6, Canada
}

\begin{abstract}
We present preliminary results of Sitelle observations of the M1-67 nebula surrounding the Wolf-Rayed stars WR 124.
\end{abstract}

Keywords. stars: Wolf-Rayet, stars: winds, outflows, ISM: individual (M1-67), ISM: bubbles

\section{The M1-67 Nebula}

M1-67, which surrounds the WN8h star WR 124, consist of N-enhanced and O-deficient gas (Esteban et al. 1991) with mixed-in dust (Vamvatira-Nakou et al. 2016). Sirianni et al. (1998) found that it consist of an expanding shell $(46 \mathrm{~km} / \mathrm{s})$ and a bipolar outflow $(88 \mathrm{~km} / \mathrm{s})$. The nebula was interpreted by Van der Sluys \& Lamers (2003) as an LBV outburst interacting with the bow shock caused by the high velocity of this runaway star.

\section{Sitelle Observations}

In May 2016, we obtained a data cube of M1-67 using the optical imaging Fourier transform spectrograph Sitelle. This instrument, installed on the CFHT, covers a wavelength range from 350 to 900 $\mathrm{nm}$ with a field of view is $11^{\prime \prime} \times 11^{\prime \prime}$ (with $0.32^{\prime \prime}$ pixels). In Figure 1, the $\mathrm{H} \alpha$ flux is shown in red and the [NII] 26548,6584 flux in blue. Two different structures can clearly be seen: a NE-SW oval shape region where hydrogen is dominant (bottom) and a NW-SE filamentary ring in which nitrogen dominates (top).

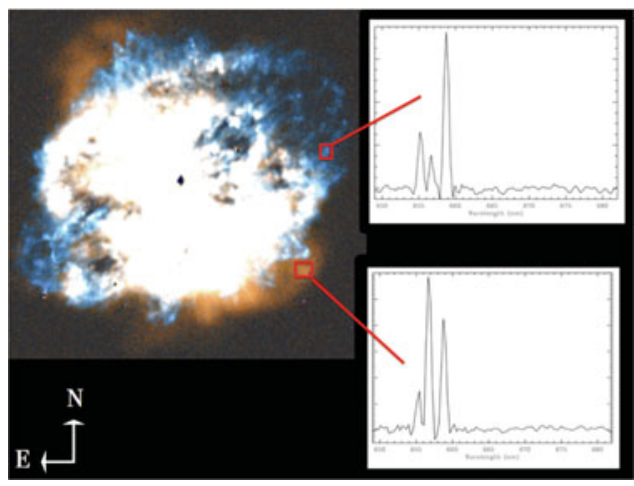

Figure 1. Sitelle line-map images of M1-67. Red is $\mathrm{H} \alpha$, Blue is [NII].

This data will provide density, temperature and abundances for the entire nebula and allow us to kinematically separate the different components of the expanding gas. This will allow us to shed new light on the past evolutionary phases undergone by the star.

\section{References}

Esteban, C., Vilchez, J. M., Manchado, A., \& Smith, L. J. 1991, A\& A, 205

Sirianni, M., Nota, A., Pasquali, A., \& Clampin, M. 1998, A\& A, 335, 1089

Vamvatira-Nakou, et al. 2016, A\& A, 588, A92

Van der Sluys, M. V. \& Lamers, H. J. G.. L. M.. 2003, A\& A, 398, 181 